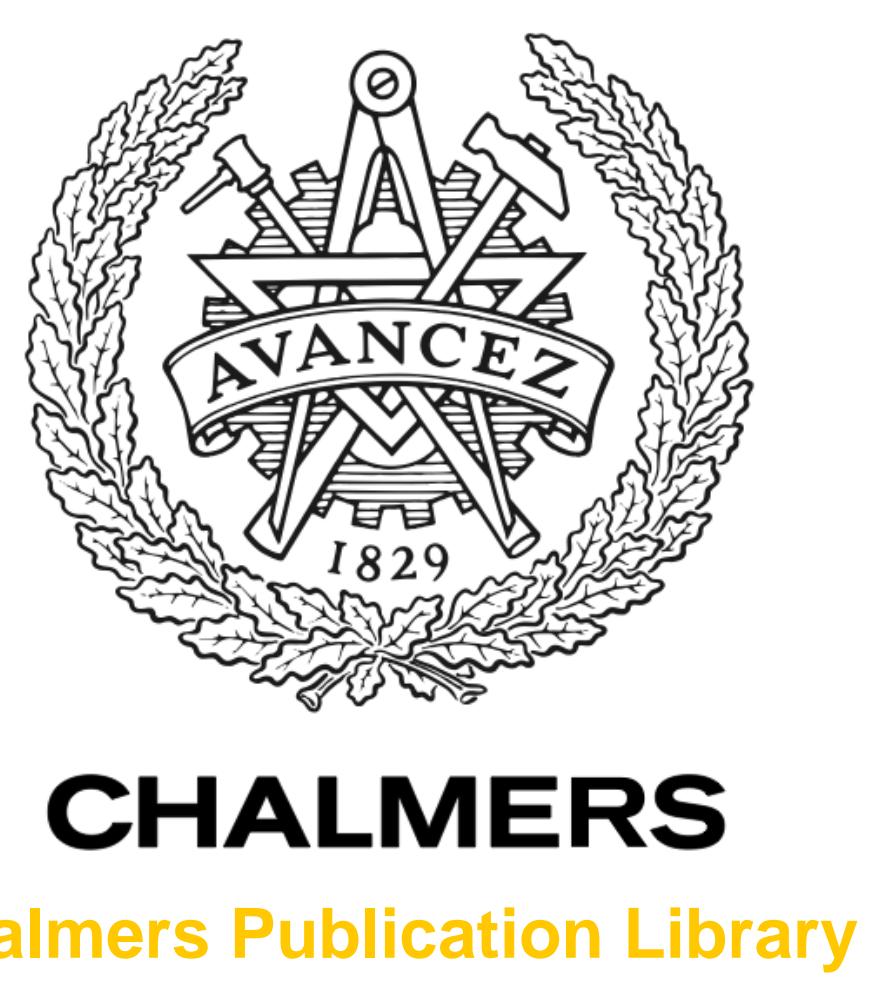

Chalmers Publication Library

\title{
Generalized pulse-position modulation for optical power-efficient communication
}

This document has been downloaded from Chalmers Publication Library (CPL). It is the author's version of a work that was accepted for publication in:

37th European Conference on Optical Communication and Exhibition, ECOC 2011, Geneva, 18 September 2011 through 22 September 2011

Citation for the published paper:

Karlsson, M. ; Agrell, E. (2011) "Generalized pulse-position modulation for optical powerefficient communication". 37th European Conference on Optical Communication and

Exhibition, ECOC 2011, Geneva, 18 September 2011 through 22 September 2011

http://dx.doi.org/10.1364/ECOC.2011.Tu.6.B.6

Downloaded from: http://publications.lib.chalmers.se/publication/150995

Notice: Changes introduced as a result of publishing processes such as copy-editing and formatting may not be reflected in this document. For a definitive version of this work, please refer to the published source. Please note that access to the published version might require a subscription.

Chalmers Publication Library (CPL) offers the possibility of retrieving research publications produced at Chalmers University of Technology. It covers all types of publications: articles, dissertations, licentiate theses, masters theses, conference papers, reports etc. Since 2006 it is the official tool for Chalmers official publication statistics. To ensure that Chalmers research results are disseminated as widely as possible, an Open Access Policy has been adopted.

The CPL service is administrated and maintained by Chalmers Library. 


\title{
Generalized Pulse-Position Modulation for Optical Power-Efficient Communication
}

\author{
Magnus Karlsson (1) and Erik Agrell (2) \\ (1) Department of Microtechnology and Nanoscience, \\ (2) Department of Signals and Systems, \\ Chalmers University of Technology, SE-412 96 Göteborg, Sweden \\ email:magnus.karlsson@chalmers.se
}

\begin{abstract}
A family of modulation formats is derived by combining pulse-position modulation (PPM) with multilevel dual-polarization signal constellations. With 16-PPM, gains of up to $5.4 \mathrm{~dB}$ are obtained over dual-polarization QPSK, at the cost of reduced spectral efficiency.
\end{abstract}

OCIS codes: (060.4080) Modulation; (060.4510) Optical communications

\section{Introduction}

Fiber-optic coherent transmission technologies are becoming increasingly popular for a number of reasons, the most important being (i) increased spectral efficiency, which comes from a practical use of multilevel and multidimensional modulation [1,2] and (ii) increased receiver sensitivities, stemming from the coherent optical receiver, which can detect both amplitude and phase $[1,10]$. With these technological breakthroughs, the system performance falls back on issues that may seem well known and textbook-like, such as the choice of modulation format. However, since the symbol space can have four, or as we will see below even more dimensions, the choice of modulation scheme deserves an elaborate discussion.

If the constellation space is limited to four dimensions (4d), we recently showed $[3,4]$ that the most power-efficient format is obtained by transmitting quadrature phase-shift keying (QPSK) data in one of two orthogonal polarization states at each time, which yields the so-called polarization-switched QPSK (PSQPSK). The corresponding signal constellation is the $4 \mathrm{~d}$ cross-polytope. For asymptotically low bit-error rate (BER), it has an asymptotic power efficiency (APE) of $\gamma=1.76 \mathrm{~dB}$. The APE equals the sensitivity gain over the conventional QPSK at very low BERs, and at a BER of $10^{-3}$, the gain reduces to $1 \mathrm{~dB}$, as was recently verified experimentally [5].

There are a number of ways of increasing the sensitivity further (without resorting to improved hardware), and they all involve increasing the dimensionality of the constellation. Additional dimensions can be obtained by transmitting dependent signals (i.e., coding) over several time slots or frequency bands. Then each time slot or frequency band will provide a new degree of freedom in which we can, in principle, modulate $4 \mathrm{~d}$ signals. If we use $K$ such subsequent time slots, we will thus have a $4 K$-dimensional signal space. Furthermore, if only one of the $K$ slots is selected for modulation, and the rest are kept powerless, we have an example of generalized pulse-position modulation (PPM). PPM was discussed for optical lines already in [6] and more recently in $[7,8]$, and it is known to have good (unbounded) sensitivity as $K$ increases, however at the expense of spectral efficiency. For finite $K$, there are interesting trade-offs between spectral efficiency and sensitivity that can be explored by overlaying PPM with a conventional modulation format, as recently suggested by Liu et al. [7]. In that work, it was demonstrated that 16-PPM combined with polarization-multiplexed QPSK (PMQPSK) can obtain an APE of $\gamma=3 \mathrm{~dB}$ of increased sensitivity over regular QPSK and PMQPSK. For quantum-limited coherent receivers, the sensitivity in photons per bit can then be directly obtained from the APE and the known sensitivity for QPSK. The purpose of this paper is to evaluate and compare the APE of known power-efficient 2-dimensional (2d) and 4d formats together with PPM. There are a few surprises, e.g., that QPSK and PMQPSK will no longer have equal performance under PPM. The PSQPSK format will again show better sensitivity than other $2 \mathrm{~d}$ and $4 \mathrm{~d}$ formats with PPM.

\section{Modulation with PPM}

The comparison of modulation schemes involves in general two main parameters; the spectral efficiency $S E$ and the APE $\gamma$, which equals the (low-BER) sensitivity gain over QPSK, transmitting the same data rate. In general, these 

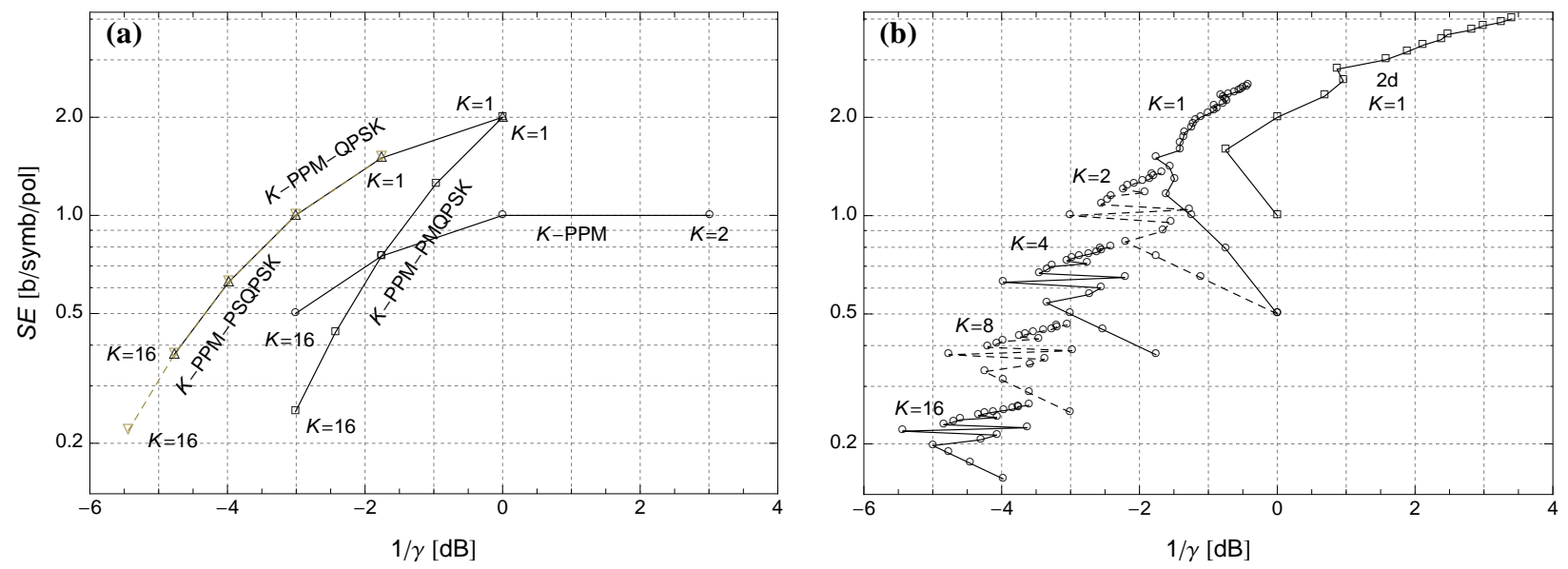

Fig. 1: (a) Chart over the asymptotic sensitivity penalty $1 / \gamma$ vs. spectral efficiency $S E$ for $K$-PPM (O), $K$-PPM-QPSK ( $\triangle$ ), $K$-PPM-PMQPSK ( $\square$ ) and $K$-PPM-PSQPSK ( $\nabla$, dashed line), where $K=1,2,4,8$, and 16. (b) Performance of $K$-PPM- $\mathscr{C}_{4, m}$ for $m=2,3, \ldots, 21$, where $\mathscr{C}_{4, m}$ is the most power-efficient $4 \mathrm{~d}$ constellations known from [4]. Included for reference are the best known $m$-ary constellations in $2 \mathrm{~d}(\square, m=2, \ldots, 16)$ and $4 \mathrm{~d}$ $(\mathrm{O}, m=2, \ldots, 32)$ without PPM, i.e., for $K=1$.

quantities are, for an $N$-dimensional, $M$-level modulation scheme given by

$$
S E=\frac{\log _{2}(M)}{N / 2}, \quad \gamma=\frac{d_{\min }^{2}}{4 \bar{E}_{s}} \log _{2}(M)
$$

where $\bar{E}_{s}$ is the average energy per symbol and $d_{\min }$ is the smallest Euclidean distance between any pair of symbols in the constellation. In symbol space, each of the $M$ symbols is placed at a distance $\sqrt{E_{s}}$ (which may vary between the symbols of the constellation) from the origin. This definition gives $S E$ in units of "bits per symbol per dimension pair," where we can often replace "dimension pair" with "polarization."

\subsection{Basic PPM}

Conventional $K$-ary PPM ( $K$-PPM) is usually assumed to use a mark with energy $E_{s}$ in one of $K$ consecutive time slots, while the rest are zero. The dimensionality is then $K$, as is the number of modulation levels, making the spectral efficiency $S E=2 \log _{2}(K) / K$. After reaching a maximum at $K=3$, the SE decreases monotonically with $K$, which is characteristic for PPM. The APE can be shown to be $\gamma=\log _{2}(K) / 2$, which is clearly unlimited with $K$. This means that PPM can have arbitrarily low sensitivities, and the price paid is spectral efficiency. This is illustrated in Fig. 1 (a), showing spectral efficiencies vs. sensitivity penalty for $K$-PPM (circles) for $K=2,4,8$, and 16.

\subsection{Generalized PPM}

An attractive way of improving $K$-PPM is, as suggested in [7], by combining it with a more efficient modulation format $\mathscr{C}$. Specifically, a symbol from a $n$-dimensional $m$-ary constellation is transmitted in one of the $K$ time slots and the zero vector in the other $K-1$ time slots. Then the total dimensionality will be $N=n K$, the number of modulation levels $M=m K$, and the spectral efficiency $S E=2 \log (m K) /(n K)$. The APE requires a bit more careful evaluation, as the minimum distance in the format can be given by the smallest of the distance within $\mathscr{C}$ and the "PPM distance," which is $\sqrt{2 E_{s}}$ for the smallest $E_{s}$ in $\mathscr{C}$. We have evaluated the $S E$ and the APE for some of the most straightforward generalizations of PPM, namely with QPSK, PSQPSK, and PMQPSK (which were demonstrated in [7]). The properties of these formats are tabulated in Table 1, and they are plotted in Fig. 1 (a) for $K=2,4,8$ and 16-PPM. It can be observed that $K$-PPM-PSQPSK, $2 K$-PPM-QPSK, and $4 K$-PPM-BPSK (not shown) are equivalent, which is not surprising given that PSQPSK, which is transmission of QPSK in one of two polarizations, is essentially the same as 2-PPM-QPSK, and analogously, QPSK is the same as 2-PPM-BPSK.

In Fig. 1 (b), $K$-PPM is combined with the previously explored 4d power-efficient formats [4]. Just as in the conventional case without PPM, the PSQSPK format turns out to have the best sensitivity. Some constellations, such as those for $m=7$ and 9, are remarkably weak with PPM. This deficiency is even more prominent for the most power-efficient $4 \mathrm{~d}$ constellations with $m \geq 22$ and $2 \mathrm{~d}$ constellations with $m \geq 5$ (not included in the figure). The reason is that these constellations have a small "PPM distance" due to a constellation point near the origin [9]. 
Table 1: Parameters of PPM and generalized PPM.

\begin{tabular}{lcccc}
\hline Format & Dimensionality $N$ & Nbr. levels $M$ & SE [b/symb/pol] & APE $\gamma$ \\
\hline \hline$K$-PPM & $K$ & $K$ & $2 \log _{2}(K) / K$ & $\log _{2}(K) / 2$ \\
$K$-PPM-QPSK & $2 K$ & $4 K$ & $\log _{2}(4 K) / K$ & $\log _{2}(4 K) / 2$ \\
$K$-PPM-PSQPSK & $4 K$ & $8 K$ & $\log _{2}(8 K) /(2 K)$ & $\log _{2}(8 K) / 2$ \\
$K$-PPM-PMQPSK & $4 K$ & $16 K$ & $\log _{2}(16 K) /(2 K)$ & $\log _{2}(16 K) / 4$ \\
\hline
\end{tabular}

\section{Discussion and Conclusions}

The paper confirms analytically the observation recently made in [7], that PPM in combination with a multilevel modulation format such as PMQPSK can achieve better sensitivities than what is possible with any uncoded (i.e., 2d or 4d) modulation format. The results in Fig. 1 and Table 1 also suggest that further improvements, in $S E$ and/or APE, are possible by replacing PMQPSK with a more power-efficient format. It is interesting to note that $K$-PPM-QPSK and $K$-PPM-PMQPSK, which are equivalent for $K=1$ (no PPM), are no longer equivalent when PPM is used. The specific example of 16-PPM at a PPM rate of 312.5 Mbaud investigated in [7] illustrates this: PMQPSK gives 8 bits per PPM symbol, thus in total $8 \times 0.3125=2.50 \mathrm{~Gb} / \mathrm{s}$. If instead PPM-QPSK were used independently in both polarizations, one would have 6 bits per polarization (2 QPSK and 4 PPM) and thus 12 bits per PPM symbol and a net bit rate of $12 \times 0.3125=3.75 \mathrm{~Gb} / \mathrm{s}$.

Another example is to compare different 16-PPM formats at the same bitrate, say $2.5 \mathrm{~Gb} / \mathrm{s}$, and using uncoded BPSK as the reference sensitivity. As shown in [7], 16-PPM-PMQPSK would require a bandwidth of $10 \mathrm{GHz}$, and gain $3 \mathrm{~dB}$ in sensitivity. Basic 16-PPM would require $10 \mathrm{GHz}$ (or using polmuxed PPM, $5 \mathrm{GHz}$ would do), while gaining $3 \mathrm{~dB}$ in sensitivity. For 16-PPM-QPSK the bandwidth requirement is $20 / 3=6.67 \mathrm{GHz}$, with a sensitivity gain of $4.8 \mathrm{~dB}$. Finally, the most sensitive format would be 16-PPM-PSQPSK, with the best sensitivity gain of $5.4 \mathrm{~dB}$ but 40/7 = 5.7 atso with the inightest bandwidth requirement of 80/7 - 11.4 GHz. These trade-offs are clearly illustrated in Fig 1 (a).

It should be noted that these sensitivity values are asymptotic, which means that slightly less improvement (especially for basic PPM which have many neighbors in signal space) can be expected at a finite BERs, such as the commonly used $10^{-3}$.

In conclusion, we have demonstrated and discussed the spectral efficiency vs. sensitivity trade-offs given by using PPM together with some standard $4 \mathrm{~d}$ modulation formats. The most sensitive $4 \mathrm{~d}$ format with PPM is PSQPSK, giving $2.4 \mathrm{~dB}$ improvement over PMQPSK when used with 16-PPM.

We wish to acknowledge funding from Vinnova within the IKT grant and the Swedish Strategic Research Foundation (SSF). We also acknowledge inspiring discussions with colleagues within the Chalmers FORCE center.

\section{References}

1. H. Sun, K.-T. Wu, and K. Roberts,"Real-time measurements of a $40 \mathrm{~Gb} / \mathrm{s}$ coherent system," Opt. Exp., 16, pp. 873-879 (2008).

2. P. J. Winzer, A. H. Gnauck, C. R. Doerr, M. Magarini, and L. L. Buhl, "Spectrally efficient long-haul optical networking using 112-Gb/s polarization-multiplexed 16-QAM," J. Lightw. Technol., 28, pp. 547-556 (2010).

3. M. Karlsson and E. Agrell, "Which is the most power-efficient modulation format in optical links?," Opt. Exp., 17, pp. 10814-10819 (2009).

4. E. Agrell and M. Karlsson, "Power-efficient modulation format in fiber optical links," J. Lightw. Technol., 27, pp. 5115-5126 (2009).

5. M. Sjödin, P. Johannisson, H. Wymeersch, P. A. Andrekson, and M. Karlsson, "Comparison of polarization-switched QPSK and polarization-multiplexed QPSK at $30 \mathrm{Gbit} / \mathrm{s}, "$ Opt. Exp., 19, pp. 7839-7846 (2011).

6. J. Pierce, "Optical channels: Practical limits with photon counting," IEEE Trans. Comm., 26, pp. 1819-1821 (1978).

7. X. Liu, T. H. Wood, R. W. Tkach, and S. Chandrasekhar, "Demonstration of record sensitivity in an optically pre-amplified receiver by combining PDM-QPSK and 16-PPM with pilot-assisted digital coherent detection," OFC'11, paper PDPB1 (2011).

8. N. W. Spellmeyer, S. L. Bernstein, D. M. Boroson, D. O. Caplan, A. S. Fletcher, S. A. Hamilton, R. J. Murphy, M. Norvig, H. G. Rao, B. S. Robinson, S. J. Savage, R. T. Schulein, M. L. Stevens, and J. P. Wang, "Demonstration of multi-rate thresholded preamplified 16-ary pulse-position-modulation," OFC'10, paper OThT5 (2010).

9. M. Karlsson and E. Agrell, "Power efficient modulation schemes," in Impact of Nonlinearities on Fiber Optic Communication (ed. S. Kumar). Springer, to appear (2011).

10. E. Ip, A. P. T. Lau, D. J. F. Barros, and J. M. Kahn, “Coherent detection in optical fiber systems,” Opt. Exp., 16, pp. $753-791$ (2008). 